\title{
Comparação de restrições de consecutividade de aulas na geração de grades horárias usando programação inteira
}

\author{
João Pedro de Sá Moreira* Glauber Renato Colnago* \\ José Roberto Colombo Junior** \\ * IFSP - Instituto Federal de Educação, Ciência e Tecnologia do \\ Estado de São Paulo. Rua Maria Cristina, 50 - Casqueiro, 11533-160, \\ Cubatão, SP, Brasil. \\ (e-mail: jopmoreira20@gmail.com; glauber.colnago@ifsp.edu.br) \\ **IFSP - Instituto Federal de Educação, Ciência e Tecnologia do \\ Estado de São Paulo. Rodovia Presidente Dutra km 145 - Jardim \\ Diamante, 12223-201, São José dos Campos, SP, Brasil \\ (e-mail: colombo.junior@ifsp.edu.br)
}

\begin{abstract}
The manual creation of timetables in educational institutions is an exhausting and prolonged activity. Course coordinators must create timetables that at the same time respect all constraints imposed by the institution while seeking to meet teacher availability preferences, for example. It becomes possible to create an optimization problem to generate the timetables automatically, reducing efforts, saving time and obtaining solutions of better quality. One of the characteristics that must be present in the solution is the consideration that the meetings must be allocated in a consecutive way. The present article aims to present an integer programming model for timetables with classrooms and subjects beforehand allocated to professors, so the optimization will be with subjects in time slots and with two different approaches for consecutiveness constraints. The approaches were tested in a model based on the one presented in order to compare its performances. The tests were conducted on CPLEX 12.8 and as a conclusion, the sets are effective for different situations, one being better applied if all divisions of subjects were previously defined while the other when these divisions can be chosen by the optimizer.

Resumo: A criação manual de grades horárias em instituições de ensino é uma atividade desgastante e prolongada. Coordenadores de curso devem criar grades que ao mesmo tempo respeitem todas as restrições impostas pela instituição enquanto buscam atender as preferências de horários de professores, por exemplo. Torna-se cabível a criação de um problema de otimização para gerar as grades horárias automaticamente, reduzindo esforços, economizando tempo e obtendo soluções de melhor qualidade. Uma das características da solução deve ser a consideração de que as aulas devem ser alocadas de forma consecutiva. O presente artigo busca apresentar um modelo de programação inteira para grades horárias com salas e disciplinas previamente atreladas aos professores, restando apenas a alocação de disciplinas em horários com duas diferentes abordagens de restrições para consecutividade de aulas. As abordagens foram testadas em um modelo baseado no apresentado visando comparar os seus desempenhos. Os testes foram feitos no CPLEX 12.8 e como conclusão, os conjuntos são efetivos para situações diferentes, um sendo melhor aplicado se todas as divisões de disciplinas forem previamente definidas enquanto o outro quando estas divisões puderem ficar a cargo do otimizador.
\end{abstract}

Keywords: optimization; integer programming; timetable, constraints.

Palavras-chaves: otimização; programação inteira; grade horária; restrições. 


\section{INTRODUÇÃO}

A construção de grades horárias é uma atividade que ocorre periodicamente em instituições de ensino de qualquer nível. Quando este processo é feito manualmente, requer um grande esforço das pessoas envolvidas na tarefa. Conforme o número de professores, disciplinas e salas aumenta, mais difícil se torna resolver o problema. Em especial, pelo fato de tratar-se de um problema NP-hard (Babaei et al., 2015), a dificuldade aumenta exponencialmente com o número de variáveis. Por esta atividade ser de extrema importância para o desenrolar dos períodos letivos, torna-se cabível a implementação de uma solução computacional, reduzindo esforços manuais e economizando tempo. Uma das possíveis soluções recai na criação de um problema de otimização para geração das grades, e neste tipo de problema, uma consideração que deve ser implementada é a organização das aulas de forma consecutiva, ou seja, dado um conjunto de aulas de uma disciplina, estas devem ocorrer de forma consecutiva na grade seguindo algum tipo de divisão.

Na Tabela 1 há um exemplo de consecutividade para duas disciplinas de 3 aulas cada. Vale ressaltar que existem diversos outros tipos de divisões dependendo do número de aulas da disciplina. A consecutividade é uma das considerações feitas para se obter uma grade de qualidade aceitável e a sua implementação pode ser feita por grupos de restrições. Neste artigo dois possíveis grupos serão apresentados.

Tabela 1. Exemplo de consecutividade de aulas

\begin{tabular}{cc} 
Sem consecutividade & Com consecutividade \\
\hline Cálculo I & Cálculo I \\
Programação I & Cálculo I \\
Cálculo I & Cálculo I \\
Programação 1 & Programação I \\
Cálculo I & Programação I \\
Programação I & Programação I \\
\hline
\end{tabular}

Proveniente da categoria de problemas de scheduling (Moreira, 2008), o problema da geração de horários, formalmente categorizado como timetable problem (Kadam, 2016) pode ser tratado como um problema de otimização combinatória (Kazarlis et al., 2005), que consiste em alocar aulas para horários e salas, respeitando uma série de restrições. Dentre as restrições mais importantes e gerais encontram-se:

(1) Garantir que a carga horária alocada das disciplinas esteja completa;

(2) Garantir que cada professor seja alocado em uma aula por vez;

(3) Garantir que cada turma tenha aula com 1 professor por vez;

(4) Limitar a carga diária e semanal de aulas de uma disciplina para uma turma;

(5) Contemplar consecutividade de aulas.

Conforme apontado por Babaei et al. (2015), vários autores investigaram esse problema e propuseram soluções. Na literatura pode-se encontrar abordagens usando modelos de programação inteira, como proposto por Srinivasan (2011) para a educação escolar, ou da Silva Kotsko et al. (2003) que aplicam especificamente para os níveis funda- mental e médio de uma escola no Estado do Paraná, ou ainda, uma abordagem usando algoritmo genético, como em Dagade et al. (2015), entre outras metodologias. Em nível superior de ensino, os problemas costumam ficar cada vez maiores, sendo formalmente alocados em uma categoria um pouco mais específica, a de University Course Timetable Problem (UCTP) (Bucco et al., 2017). Dentre os trabalhos na área, destaca-se o de Daskalaki et al. (2004), que apresenta um modelo consistente para aplicações em universidades e que foi utilizado como base para a formulação de um dos grupos de restrições de consecutividade tratados neste artigo. Para este modelo, os professores e as salas são atribuídas previamente para as disciplinas.

O modelo discutido neste artigo foi construído com foco nas particularidades encontradas no Instituto Federal de Educação, Ciência e Tecnologia de São Paulo (IFSP) Campus Cubatão (Moreira, 2018) e tem como ponto de partida as restrições apresentadas anteriormente. Aqui buscar-se-á uma análise a respeito das restrições de consecutividade aplicadas neste modelo e como estas se comparam com um conjunto de restrições de consecutividade baseado no trabalho de Daskalaki et al. (2004).

Nota-se que não são todos os trabalhos na literatura que abordam a necessidade de contemplar aulas consecutivas. Feng et al. (2017) apresenta um levantamento a respeito de 33 trabalhos relacionados ao tema de otimização de grades horárias, e destes, apenas 9 trabalhos possuem alguma forma de envolver consecutividades nas grades. E é evidente que a garantia de consecutividade nas aulas melhora substancialmente a qualidade da grade horária. Portanto, este trabalho segue esta vertente, focando na discussão a respeito de formas de garantir diferentes tipos de consecutividades.

O restante deste artigo está organizado da seguinte forma: na Seção 2 é apresentado o problema de otimização, na Seção 3 são apresentadas as simulações feitas e por fim, a Seção 4 apresenta as conclusões do artigo.

\section{FORMULAÇÃO DO PROBLEMA DE OTIMIZAÇÃO}

Primeiramente serão apresentados os conjuntos utilizados na formulação. O primeiro deles é o conjunto $\mathbb{P M C}$ que agrupa tuplas para cada atribuição de aula. Cada elemento deste conjunto é formado por uma associação de um professor $p \in \mathbb{P R O F}$, que ministra uma disciplina $m \in$ $\mathbb{D I S C}$, em uma turma $c \in \mathbb{T} U \mathbb{R} M A$.

- Conjunto de atribuição de aulas: $\mathbb{P M C}=\{$ professor $\boldsymbol{p}$, disciplina $\boldsymbol{m}$, turma $\boldsymbol{c}\}$

Cada possível dia de aula faz parte do conjunto $\mathbb{D} \mathbb{I}$.

- Conjunto de dias da semana: $\mathbb{D I A}=\{2,3,4, \ldots, 7\}$

Cada professor possui uma série de preferências de horários e estes dados podem ser agrupados em um conjunto.

- Conjunto de preferências de professores: $\mathbb{P R \mathbb { R }}=$ $\{$ professor $\boldsymbol{p}$, dia $\boldsymbol{d}$, horário $\boldsymbol{h}$, turno $\boldsymbol{t}\}$

O conjunto $\mathbb{H} \mathbb{O} \_\mathbb{T} U \mathbb{R}$ associa possíveis horários de aula com o turno em que ocorrem. 
- Conjunto de associação entre horários de aula e turno: $\mathbb{H O R} \mathbb{T} \mathbb{R}=\{$ horário $\boldsymbol{h}$, turno $\boldsymbol{t}\}$

Neste exemplo serão admitidos dois turnos (vespertino e noturno) com disciplinas que tem até 5 aulas por dia. Porém o material apresentado aqui pode ser estendido para situações diferentes da considerada.

A variável principal que determina a combinação que configura uma aula é a variável $x$, definida por:

$$
x_{p m c d h t}= \begin{cases}1, & \begin{array}{l}
\text { se o professor } \mathbf{p} \text { dá aula para a } \\
\text { disciplina } \mathbf{m}, \text { na turma } \mathbf{c} \\
\text { no dia } \mathbf{d}, \text { horário } \mathbf{h} \text { e turno } \mathbf{t}
\end{array} \\
0, & \text { caso contrário }\end{cases}
$$

O uso de uma variável auxiliar é necessário para que seja possível ter um controle dos dias em que uma disciplina ocorre:

$$
y_{m d}= \begin{cases}1, & \begin{array}{l}
\text { se o professor } \mathbf{p} \text { dá aula para a } \\
\text { disciplina } \mathbf{m}, \text { no dia } \mathbf{d}
\end{array} \\
0, & \text { caso contrário }\end{cases}
$$

A função objetivo maximiza a alocação de aulas que atenda as preferências de disponibilidade dos professores, enquanto busca agrupar aulas nos inícios dos turnos e minimiza a quantidade de aulas alocadas aos sábados.

$$
\begin{aligned}
& \text { Max pref }=\sum_{(p, m, c) \in \mathbb{P M C}} \sum_{d \in \mathbb{D I} \mathbb{A}} \sum_{(h, t) \in \mathbb{H} \mathbb{O} \_\mathbb{T} \cup \mathbb{R}} x_{p m c d h t} \\
& \mid(p, d, h, t) \in \mathbb{P R} \mathbb{E} \mathbb{F}
\end{aligned}
$$

$$
-\sum_{(p, m, c) \in \mathbb{P M C} \mathbb{C}} \sum_{d \in \mathbb{D I} \mathbb{A}} 0,01 * x_{p m c d(h=4)(t=2)}+0,1 * x_{p m c d(h=5)(t=2)}
$$

$$
-2 * \sum_{(p, m, c) \in \mathbb{P M C} \mathbb{c}} \sum_{\substack{(h, t) \in \mathbb{H} \mathbb{R} \mathbb{T} \cup \mathbb{R} \\ \mid c \in \mathbb{T} \mathbb{S A B}}} x_{p m c(d=7) h t}
$$

A parcela da função objetivo em (4) minimiza a alocação de aulas nos dois últimos horários, ou seja, se houver a necessidade de manter alguma aula vaga, a tendência será alocá-la no fim do turno, permitindo a liberação dos alunos mais cedo. A parcela em (5) minimiza a alocação de aulas aos sábados. Como não são todas as turmas que têm aulas neste dia, a parcela itera apenas sobre as turmas pertencentes ao conjunto $\mathbb{T} \_\mathbb{S} A \mathbb{B}$ (Turmas com aulas aos sábados). As restrições básicas apresentadas nos itens de (1) - (5) na Seção 1 serão formuladas matematicamente a seguir.

1. Garantir que a carga horária alocada das disciplinas esteja completa:

$$
\sum_{(p, m, c) \in \mathbb{P M C}} \sum_{\substack{d \in \mathbb{D I} \mathbb{A} \\ \forall m \in \mathbb{D}}} \sum_{\substack{(h, t) \in \mathbb{H} \mathbb{R} \mathbb{R} \mathbb{C} \\ \forall m \mathbb{R}}} x_{p m c d h t}=Q t d_{m}
$$

onde o parâmetro $Q t d_{m}$, é um valor inteiro igual a quantidade de aulas que cada disciplina $\mathbf{m}$ deve ter ao longo da semana.

2. Garantir que cada professor seja alocado em até uma aula por vez:

$$
\sum_{(p, m, c) \in \mathbb{P M C}} x_{p m c d h t} \leq 1
$$

$$
\forall p \in \mathbb{P R O F E S S O R , ~} \forall d \in \mathbb{D I} \mathbb{A}, \forall(h, t) \in \mathbb{H} \mathbb{O} \mathbb{R} \_\mathbb{T} \mathbb{R}
$$

3. Garantir que cada turma tenha aula com até 1 professor por vez:

$$
\begin{gathered}
\sum_{(p, m, c) \in \mathbb{P M C}} x_{p m c d h t} \leq 1 \\
\forall c \in \mathbb{T} \mathbb{\mathbb { R } M} \mathbb{A}, \forall d \in \mathbb{D} \mathbb{I} \mathbb{A}, \forall(h, t) \in \mathbb{H} \mathbb{O} \mathbb{R} \_\mathbb{T} \mathbb{R}
\end{gathered}
$$

4. Limitar a carga diária e semanal de aulas de uma disciplina para uma turma.

$$
\begin{gathered}
\sum_{h} x_{p m c d h t} \leq Q M a x_{m} \\
\forall(p, m, c) \in \mathbb{P M} \mathbb{C}, \forall d \in \mathbb{D I} \mathbb{A}, \forall(h, t) \in \mathbb{H} \mathbb{O} \_\mathbb{T} \mathbb{R} \\
y_{m d} \geq x_{p m c d h t} \\
\forall(p, m, c) \in \mathbb{P M C}, \forall d \in \mathbb{D} \mathbb{A}, \forall(h, t) \in \mathbb{H} \mathbb{O} \mathbb{R}_{-} \mathbb{T} U \mathbb{R} \\
\sum_{d \in \mathbb{D} \mathbb{A}} y_{m d} \leq S M a x_{m} \\
\forall(p, m, c) \in \mathbb{P M} \mathbb{C}, \forall(h, t) \in \mathbb{H} \mathbb{O} \_\mathbb{T} \mathbb{R}
\end{gathered}
$$

A restrição (9) garante que, para um determinado dia, a soma de todos os horários de aulas para uma disciplina seja menor ou igual ao parâmero $Q M a x_{m}$. A restrição (10) contabiliza os dias em que uma disciplina tenha no mínimo 1 aula, enquanto a restrição (11) limita a ocorrência semanal da disciplina através de um parâmetro $S M a x_{m}$. Os parâmetros $Q \operatorname{Max}_{m}$ e $S \operatorname{Max}_{m}$ são determinados de modo a garantir a distribuição adequada dos blocos de aula, conforme será visto a seguir.

\section{Contemplar consecutividade de aulas}

São estudadas e apresentadas a seguir duas abordagens de restrições para garantir a consecutividade.

Definição formal do primeiro grupo de restrições de consecutividade

Nesta formulação, deve-se definir previamente como as aulas de uma disciplina serão divididas ao longo da semana e para cada tipo de divisão, utiliza-se um grupo de restrições. É importante salientar que o otimizador não terá liberdade para decidir como ocorrerão as divisões, já que estas são estabelecidas antes do processo de otimização. Por exemplo, considere que uma determinada disciplina com 5 aulas será dividida em dois dias, um com 2 aulas e outro com 3 aulas (entende-se esta divisão como $2+3$ aulas). Nesse caso, é necessário $\operatorname{SMax}_{m}=2$.

Para cada tipo de divisão, deve existir um conjunto de disciplinas associado. Na Tabela 2, são apresentadas restrições para divisões de aulas de disciplinas que ocorrem 
Tabela 2. Restrições para cada divisão considerada $(\forall(p, m, c) \in \mathbb{P M} \mathbb{C}, \forall d \in \mathbb{D} \mathbb{I}, \forall(h, t) \in$ $\left.\mathbb{H} O \mathbb{R} \_\mathbb{T} U \mathbb{R}\right)$.

\begin{tabular}{|c|c|c|c|}
\hline Número de aulas & Qtd. de aulas consecutivas na semana (divisão) & Restrições & Condições \\
\hline $2,4,3$ & $2,2+2,2+1$ & $\begin{array}{l}x_{\text {pmcdht }}+x_{\text {pmcd }(h+2) t} \leq 1 \\
x_{\text {pmcdht }}+x_{\text {pmcd }(h+3) t} \leq 1 \\
x_{\text {pmcdht }}+x_{\text {pmcd }(h+4) t} \leq 1 \\
x_{\text {pmcdht }}+x_{\text {pmcd }(h+5) t} \leq 1\end{array}$ & $\begin{array}{l}h \leq 4 \\
h \leq 3 \\
h \leq 2 \\
h=1\end{array}$ \\
\hline 3 & 3 & $\begin{array}{l}x_{\text {pmcdht }}+x_{\text {pmcd }(h+3) t} \leq 1 \\
x_{\text {pmcdht }}+x_{\text {pmcd }(h+4) t} \leq 1 \\
x_{\text {pmcdht }}+x_{\text {pmcd }(h+5) t} \leq 1\end{array}$ & $\begin{array}{l}h \leq 3 \\
h \leq 2 \\
h=1\end{array}$ \\
\hline 4 & 4 & $\begin{array}{l}x_{\text {pmcdht }}+x_{\text {pmcd }(h+4) t} \leq 1 \\
x_{\text {pmcdht }}+x_{\text {pmcd }(h+5) t} \leq 1\end{array}$ & $\begin{array}{l}h \leq 2 \\
h=1\end{array}$ \\
\hline 5 & $3+2$ & $\begin{array}{l}2 x_{p m c d h t}-x_{p m c d(h+1) t}+2 x_{p m c d(h+2) t} \leq 3 \\
2 x_{p m c d h t}+x_{p m c d(h+2) t}+2 x_{p m c d(h+3) t} \leq 3 \\
2 x_{p m c d h t}+x_{p m c d(h+3) t}+2 x_{p m c d(h+4) t} \leq 3 \\
2 x_{p m c d h t}+x_{p m c d(h+4) t}+2 x_{p m c d(h+5) t} \leq 3\end{array}$ & $\begin{array}{l}h \leq 4 \\
h \leq 3 \\
h \leq 2 \\
h=1\end{array}$ \\
\hline
\end{tabular}

em até 5 aulas. A variável $y_{m d}$ deverá ser empregada para cada tipo de divisão, dependendo da quantidade de dias requeridos. Quando é necessário manter 5 aulas ocorrendo de forma consecutiva, não é necessário utilizar uma restrição de consecutividade para isto, basta empregar a restrição (10) e a (11) sendo o parâmetro $S M a x_{m}$ desta disciplina igual a 1 (para disciplinas com no máximo 5 aulas).

Para disciplinas com apenas uma aula, nenhuma restrição precisa ser aplicada, e para divisões do tipo $1+1+\ldots+1$, ou seja, distribuindo uma aula por dia, só é necessário empregar (10) e (11) sendo o parâmetro $\operatorname{SMax}_{m}$ da disciplina igual ao número de de aulas requeridas.

Na primeira linha da Tabela 2, o conjunto de restrições serve para os três casos representados, porém o que irá variar será a forma como as restrições (10) e (11) são empregadas.

Definição formal do segundo grupo de restrições de consecutividade

Esta outra abordagem é baseada em Daskalaki et al. (2004), e garante que as aulas, quando alocadas, serão consecutivas. Nesse caso, o otimizador possui a liberdade de escolher qual é a divisão que provê um melhor valor de função objetivo. É possível forçar a divisão de uma determinada disciplina em algum número de dias com os parâmetros $Q \operatorname{Max}_{m}$ e $S M a x_{m}$.

Este grupo de restrições não permite aulas únicas, se isto deve ocorrer para alguma disciplina, esta não deve ser inserida no modelo com estas restrições.

Com $(p, m, c) \in \mathbb{P M C}$ e $d \in \mathbb{D} \mathbb{I}$, as restrições que garantem a consecutividade são apresentadas a seguir.

$$
\begin{gathered}
x_{p m c d h t}-x_{p m c d(h+1) t} \leq 0 \\
(h, t) \in \mathbb{H} \mathbb{O} \_\mathbb{T} \mathbb{R} \mid h=t=1 \\
-x_{p m c d h t}+x_{p m c d(h+1) t}-x_{p m c d(h+2) t} \leq 0 \\
(h, t) \in \mathbb{H} \mathbb{O} \_\mathbb{T} \mathbb{R} \mid 1 \leq h \leq 4 e t=1 \\
-x_{p m c d(h+3) t}+x_{p m c d(h+4) t} \leq 0 \\
(h, t) \in \mathbb{H} \mathbb{O} \mathbb{R} \mathbb{R} \mid h=t=1
\end{gathered}
$$

$$
\begin{gathered}
-x_{p m c d(h+1) t}+x_{p m c d(h+2) t}-x_{p m c d(h+3) t}-1 \leq 0 \\
(h, t) \in \mathbb{H} \mathbb{O} \mathbb{R} \mathbb{R} \mid h=t=1
\end{gathered}
$$

Para aplicação em períodos com mais (ou menos) de 5 aulas, basta modificar ou adicionar restrições no grupo de modo adequado utilizando a mesma lógica para evitar quebra na consecutividade.

Existe a possibilidade de uma turma possuir disciplinas com uma distribuição de aulas que torna o problema infactível dependendo de qual for a divisão de aulas escolhida. O exemplo seria uma turma que possui 5 dias de aula, cada um com 5 aulas possíveis e a turma possui 8 disciplinas de 3 aulas cada. Seguindo o padrão, seriam empregadas as divisões do tipo 3 aulas consecutivas para cada disciplina, o que torna o problema infactível pois essa divisão não é possível para todas as 8 disciplinas nos 5 horários possíveis em 5 dias.

No primeiro caso, basta ter modelado o conjunto de restrições que contempla uma divisão diferenciada e aplicar este conjunto nesta turma. No exemplo dado, inserindo 3 disciplinas da turma no conjunto de restrições para divisão 3 aulas em $2+1$, resolve-se o problema. Já no segundo caso, o problema pode ser tratado com o uso de uma variável auxiliar que serve para desativar ou ativar a consecutividade em um dia. É uma forma de relaxar a restrição quando houver necessidade. Em ambas as situações, o ideal é que estes casos sejam previamente identificados e recebam este tratamento especial. Embora este tratamento não tenha sido considerado para os testes neste trabalho, o segundo conjunto de restrições, agora modificado com a variável auxiliar é apresentado em (16) (19). E deve-se adicionar a parcela (20) na função objetivo.

$$
\begin{gathered}
x_{p m c d h t}-x_{p m c d(h+1) t} \leq a_{p m c d} \\
(h, t) \in \mathbb{H} \mathbb{O} \_\mathbb{T} \mathbb{R} \mid h=t=1 \\
-x_{p m c d h t}+x_{p m c d(h+1) t}-x_{p m c d(h+2) t} \leq a_{p m c d} \\
(h, t) \in \mathbb{H} \mathbb{O} \_\mathbb{T} \mathbb{R} \mid 1 \leq h \leq 4 \text { e } t=1 \\
-x_{p m c d(h+3) t}+x_{p m c d(h+4) t} \leq a_{p m c d} \\
(h, t) \in \mathbb{H} \mathbb{O} \_\mathbb{T} \mathbb{R} \mid h=t=1
\end{gathered}
$$




$$
\begin{gathered}
-x_{p m c d(h+1) t}+x_{p m c d(h+2) t}-x_{p m c d(h+3) t}-1 \leq a_{p m c d} \\
(h, t) \in \mathbb{H} \mathbb{O R} \mathbb{R} \mathbb{R} \mid h=t=1
\end{gathered}
$$

$$
-10000 * \sum_{(p, m, c) \in \mathbb{P M C}} \sum_{d \in \mathbb{D} \mathbb{I} \mathbb{A}} a_{p m c d}
$$

\section{EXEMPLOS NUMÉRICOS}

Uma vez que as restrições foram devidamente definidas, pode-se excursionar sobre as simulações realizadas com o intuito de comparar os seus funcionamentos. Foi simulada uma instância com 10 turmas do período noturno (com algumas aulas ocorrendo aos sábados no período vespertino). Foram consideradas todas as restrições básicas anteriormente apresentadas assim como algumas outras restrições específicas para adequar o modelo ás práticas padrões da instituição. O solver utilizado foi o ILOG CPLEX Optimization Studio 12.8.0.0 - versão acadêmica - em uma máquina com Intel Core I5 7200U e 8 GB de RAM. A linguagem utilizada para a implementação do modelo foi a Optimization Programming Language (OPL).

O IBM ILOG Cplex Optimization Studio utiliza um algoritmo baseado no método de Branch-and-Cut, que se trata de um método exato que consiste na combinação do método de planos de corte e Branch-and-Bound (Mitchell, 2002). A qualidade das soluções foi comparadas através do valor de função objetivo, gap relativo e tempo de solução. O gap relativo é definido pela documentação como a expressão em (21).

$$
g a p=\frac{\mid \text { melhor_limitante }- \text { melhor_inteiro } \mid}{1^{-10}+\mid \text { melhor_inteiro } \mid}
$$

A expressão em (21) basicamente dimensiona a diferença relativa entre a melhor solução inteira conhecida e a melhor solução não inteira que limita possíveis soluções inteiras melhores. Quanto menor for o gap relativo, mais próxima da condição ótima a solução atual está. Podese definir um valor limite de gap para o algoritmo, de modo que ao alcançar este valor, o processo de otimização é interrompido e a solução inteira atual, salva.

Nas simulações rodadas, foram considerados dois turnos de aulas $(1 \leq t \leq 2)$ com 5 horários de aulas possíveis $(1 \leq h \leq 5)$ de segunda a sábado $(2 \leq d \leq 7)$.

Para comparação dos grupos de restrições as simulações foram realizadas de modo a utilizar a maior parte das divisões corriqueiras em instituições de ensino. Para o primeiro grupo de restrições, as turmas foram divididas de modo que ocorressem diferentes tipos de divisões (por exemplo, algumas turmas de 4 aulas foram direcionadas para divisão $2+2$ enquanto outras para 4 aulas consecutivas direto). $\mathrm{E}$, para comparar diretamente com a atuação do segundo grupo de restrições, os parâmetros de quantidade de aulas máxima diária e de grupos de aula semanais $\left(Q \operatorname{Max}_{m} \mathrm{e}\right.$ $S M a x_{m}$ ) foram modificados adequadamente para que as divisões fossem as mesmas. Dessa forma, pode-se comparar a velocidade e qualidade da solução. E por último, o segundo grupo de restrições foi testado novamente, agora com o maior nível de autonômia possível para verificar o quanto isto impacta na solução.

A Tabela 3 apresenta a relação de divisão de aulas adotadas, enquanto a Tabela 4 apresenta algumas informações sobre a instância considerada.

Tabela 3. Relação de divisões consideradas

\begin{tabular}{cc} 
Tipo de divisão & Qtd. de disciplinas \\
\hline 2 & 29 \\
3 & 26 \\
4 & 8 \\
$2+2$ & 7 \\
5 & 3 \\
$3+2$ & 4 \\
\hline Total de Disciplinas & 77 \\
\hline
\end{tabular}

Tabela 4. Instância considerada

\begin{tabular}{lc}
\hline $\mathrm{N}^{\circ}$ de turmas & 10 \\
$\mathrm{~N}^{\circ}$ de disciplinas & 77 \\
$\mathrm{~N}^{\circ}$. de professores & 43 \\
$\mathrm{~N}^{\circ}$ de váriáveis & 9321 \\
\hline
\end{tabular}

Tabela 5. Resultados da primeira simulação

\begin{tabular}{c|cc} 
& $1^{\circ}$ grupo de restrições & $2^{\circ}$ grupo de restrições \\
\hline Tempo (s) & 2,12 & 55,65 \\
Objetivo & 100,32 & 100,32 \\
Gap (\%) & 0 & 0 \\
\hline
\end{tabular}

Os resultados da primeira simulação são apresentados na Tabela 5. Para cada um dos dois casos, o modelo foi rodado três vezes e o tempo apresentado é uma média dos tempos obtidos nas simulações. Como critério de parada para o processo de otimização, esperou-se o otimizador alcançar um gap abaixo de 0.0001, considerando isto como a garantia de otimalidade alcançada. $\mathrm{O}$ valor da função objetivo neste caso serve apenas para comparar o nível de qualidade das soluções obtidas. Quanto maior o objetivo, melhor é a solução.

A segunda simulação consiste em verificar a funcionalidade do segundo grupo de restrições quando permite-se uma maior liberdade na escolha da divisão pelo otimizador. Isto significa que as disciplinas de 4 aulas terão agora até 4 aulas diárias em até 2 dias da semana, por exemplo, então o otimizador poderá escolher entre alocar 4 aulas consecutivas ou 2 aulas em 2 dias diferentes. O mesmo raciocínio se aplica a disciplinas de 5 aulas. Ao realizar as simulações, nota-se que há uma certa resistência em garantir a otimalidade da solução devido ao maior número de verificações a serem feitas. Por este motivo, será apresentada uma evolução do valor do gap obtido neste teste até alcançar o ótimo.

Tabela 6. Resultados da segunda simulação (segundo grupo de restrições)

\begin{tabular}{c|cccc}
\hline Tempo (s) & 10 & 120 & 355 & 618 \\
Objetivo & 107,02 & 107,02 & 107,02 & 107,02 \\
Gap (\%) & 10 & 5.87 & 0.47 & 0 \\
\hline
\end{tabular}

A seguir serão apresentados dois exemplos de turmas provenientes das dez escolhidas para as simulações. Os resultados apresentados na forma de grade servirão para comparar como a divisão de aulas pode mudar dependendo 
do conjuntos de restrições empregado. As tabelas (7) e (8) são referentes a primeira simulação, onde as divisões foram pré-determinadas. Já as tabelas (9) e (10) são provenientes da segunda simulação, onde o otimizador teve liberdade para determinar quais seriam as divisões mais favoráveis ao objetivo.

Tabela 7. Horário para turma 1 (simulação 1)

\begin{tabular}{|c|c|c|c|c|c|}
\hline Horário & Seg. & Ter. & Qua. & Qui. & Sex. \\
\hline $1^{\text {a }}$ Aula & Disc. 1 & Disc. 3 & Disc. 5 & Disc. 7 & Disc. 9 \\
\hline $2^{\text {a } \text { Aula }}$ & Disc. 1 & Disc. 3 & Disc. 5 & Disc. 7 & Disc. 9 \\
\hline $3^{\text {a }}$ Aula & Disc. 2 & Disc. 3 & Disc. 5 & Disc. 7 & Disc. 9 \\
\hline $4^{\text {a } \text { Aula }}$ & Disc. 2 & Disc. 4 & Disc. 6 & Disc. 8 & Disc. 9 \\
\hline $5^{\text {a } \text { Aula }}$ & Disc. 2 & Disc. 4 & Disc. 6 & Disc. 8 & Disc. 9 \\
\hline
\end{tabular}

Tabela 8. Horário para turma 1 (simulação 2)

\begin{tabular}{|c|c|c|c|c|c|}
\hline Horário & Seg. & Ter. & Qua. & Qui. & Sex. \\
\hline $1^{\text {a }}$ Aula & Disc. 2 & Disc. 3 & Disc. 6 & Disc. 7 & Disc. 4 \\
\hline $2^{\mathrm{a}}$ Aula & Disc. 2 & Disc. 3 & Disc. 6 & Disc. 7 & Disc. 4 \\
\hline $3^{\mathrm{a}}$ Aula & Disc. 2 & Disc. 3 & Disc. 5 & Disc. 7 & Disc. 9 \\
\hline $4^{\mathrm{a}}$ Aula & Disc. 9 & Disc. 1 & Disc. 5 & Disc. 8 & Disc. 9 \\
\hline $5^{\mathrm{a}}$ Aula & Disc. 9 & Disc. 1 & Disc. 5 & Disc. 8 & Disc. 9 \\
\hline
\end{tabular}

Tabela 9. Horário para turma 2 (simulação 1)

\begin{tabular}{|c|c|c|c|c|c|}
\hline Horário & Seg. & Ter. & Qua. & Qui. & Sex. \\
\hline $1^{\text {a } A u l a ~}$ & Disc. A & Disc. B & Disc. D & Disc. D & Disc. E \\
\hline $2^{\text {a Aula }}$ & Disc. A & Disc. B & Disc. D & Disc. D & Disc. E \\
\hline $3^{\text {a } A u l a ~}$ & Disc. A & Disc. C & Disc. E & Disc. F & Disc. C \\
\hline $4^{\text {a Aula }}$ & Disc. A & Disc. C & Disc. E & Disc. F & Disc. C \\
\hline $5^{\text {a Aula }}$ & - & - & - & - & Disc. C \\
\hline
\end{tabular}

Tabela 10. Horário para turma 2 (simulação 2)

\begin{tabular}{|c|c|c|c|c|c|}
\hline Horário & Seg. & Ter. & Qua. & Qui. & Sex. \\
\hline Aula $1^{\text {a }}$ & Disc. F & Disc. B & Disc. C & Disc. A & Disc. D \\
\hline Aula $2^{\text {a }}$ & Disc. F & Disc. B & Disc. C & Disc. A & Disc. D \\
\hline Aula $3^{\text {a }}$ & Disc. E & Disc. A & Disc. C & Disc. D & Disc. E \\
\hline Aula $4^{\text {a }}$ & Disc. E & Disc. A & Disc. C & Disc. D & Disc. E \\
\hline Aula $5^{\text {a }}$ & - & - & Disc. C & - & - \\
\hline
\end{tabular}

Os grupos de restrições possuem suas particularidades que podem torná-los aplicáveis em situações diferentes. Para o primeiro grupo de restrições é necessário dividir previamente as disciplinas, porém, conforme evidenciado pelos resultados, sua aplicação é mais rápida pois demanda menos verificações a serem feitas para determinar se a solução é ótima, pois o seu funcionamento se baseia em forçar a alocação na divisão escolhida. Já o segundo grupo tem menos restrições e dá autonomia para o otimizador na escolha das melhores divisões. Na Tabela 5 nota-se que o seu tempo de solução foi maior que o do primeiro grupo, essa diferença dá-se pois o segundo grupo teve que passar mais tempo até verificar a otimalidade da solução. Isso se dá por conta da restrição (9) que é um conjunto de verificações a mais que devem ser feitas para cada dia. Na segunda simulação, por ter dado uma maior gama de opções de divisões, o otimizador gasta ainda mais tempo na verificação da otimalidade, porém, nota-se que o valor do objetivo alcançado foi maior do que na simulação 1 mesmo antes do gap alcançar 0 , o que significa que aplicando um critério de parada por tempo, uma solução mais satisfatória que a de objetivo 100,32 poderia ser obtida, denotando que o otimizador encontrou uma solução melhor utilizando divisões diferentes, determinadas automaticamente e respeitando os parâmetros $Q \max _{m}$ e $S \max x_{m}$.

\section{CONCLUSÃO}

Em suma, a utilização do primeiro grupo de restrições torna-se mais adequada se todas as divisões forem previamente definidas e se o trabalho adicional na separação dos conjuntos para cada divisão não for um problema. Vale ressaltar que se estas condições não forem aceitas, a sua aplicação não é efetiva. O segundo grupo de restrições é mais adequado quando não há a necessidade da definição das divisões das disciplina, porém, mesmo se há um conhecimento sobre quais divisões devem ser feitas para uma parte da disciplinas, ou até mesmo para todas, o segundo grupo ainda é efetivo, sendo ainda melhor aplicado com um critério de parada por tempo. Vale ressaltar também que sua implementação é menos trabalhosa do que a do primeiro.

\section{REFERENCIAS}

Babaei, H., Karimpour, J., and Hadidi, A. (2015). A survey of approaches for university course timetabling problem. Computers and Industrial Engineering, 86, 43 -59 .

Bucco, G.B., Bornia-Poulsen, C.J., and Bandeira, D.L. (2017). Desenvolvimento de um modelo de programação linear para o problema da construção de grades horárias em universidades. Gestão e Produção, 24, 40 - 49.

da Silva Kotsko, E.G., Steiner, M.T.A., and da Fonseca Machado., A.L. (2003). Otimização na construção da grade horária escolar - uma aplicação -. $X X X V$ Simpósio Brasileiro de Pesquisa Operacional.

Dagade, R., Hassan, S.S., Devhare, P., Khilari, S., and Sarda, S. (2015). Timetable scheduling using genetic algorithm. Multidisciplinary Journal of Research in Engineering and Technology, October/2015, 692-696.

Daskalaki, S., Birbas, T., and Housos, E. (2004). An integer programming formulation for a case study in university timetabling. European Journal of Operational Research, 153(1), 117 - 135.

Feng, X., Lee, Y., and Moon, I. (2017). An integer program and a hybrid genetic algorithm for the university timetabling problem. Optimization Methods and Software, 32(3), 625-649.

Kadam, Vinod e Yadav, S. (2016). Academic timetable scheduling : Revisited. International Journal of Research in Science and Engineering, April/2016, 417 - 423.

Kazarlis, S., Petridis, V., and Fragkou, P. (2005). Solving university timetabling problems using advanced genetic algorithms. 5th international conference on technology and automation, october 15-16, 2005, thessaloniki, greece, proceedings of the icta, 131-136.

Mitchell, J. (2002). Branch-and-cut algorithms for combinatorial optimization problems 1. Handbook of Applied Optimization.

Moreira, J. P. e Colnago, G.R. (2018). Otimização da grade horária do ifsp - campus cubatão: dimensionando a problemática e modelando as restrições. L Simpósio Brasileiro de Pesquisa Operacional.

Moreira, J.J. (2008). A system for automatic construction of exam timetable using genetic algorithms. Revista de Estudos Politécnicos, 319 - 336.

Srinivasan, S. (2011). Modeling the Homeschool timetabling problem using Integer programming. Master's thesis, Virginia Commonwealth University. 